\title{
Using Mobile Devices to Personalize Pervasive Displays
}

\author{
Thomas Kubitza ${ }^{1,3}$, Sarah Clinch $^{1}$, Nigel Davies ${ }^{1}$, and Marc Langheinrich ${ }^{2}$ \\ ${ }^{1}$ Lancaster University, ${ }^{2}$ University of Lugano (USI) and ${ }^{3}$ University of Duisburg-Essen
}

\section{INTRODUCTION}

There is a significant body of work that has explored how content shown on public displays can be tailored for viewers in the vicinity. In [2] the authors proposed a system that detected Bluetooth devices carried by viewers in order to schedule appropriate adverts. More recently Davies et al [1] proposed the use of Bluetooth device names as a means by which users could signal their interests to nearby displays - effectively providing a unidirectional control channel between users and pervasive displays. However, power and privacy concerns mean that many users no longer leave their Bluetooth devices switched on and alternative approaches to display personalization are required. In this demonstration we show a working implementation of Tacita - a system designed to provide such an alternative approach.

\section{DEMONSTRATION OVERVIEW}

Our demonstration consists of a public display that is able to show information from a number of applications including a Weather App (Figure 1) and a News App. The content displayed by these applications can be tailored to the needs of nearby viewers. For example, viewers may choose that the Weather App shows weather information for their home town, their current location or their forthcoming vacation destination.
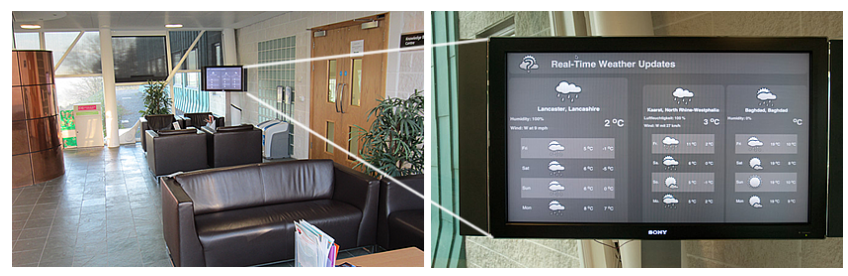

Figure 1: Weather Display App

To support personalisation our system consists of four components: 1) an Android based mobile application that lets viewers define display application preferences, 2) a display component, managed by a display owner, that schedules, renders and outputs content on a display, 3) a set of web based applications specifically designed for public displays and 4) a map provider, that provides a database of public displays, their locations and their capabilities (e.g. viewing area and available applications).
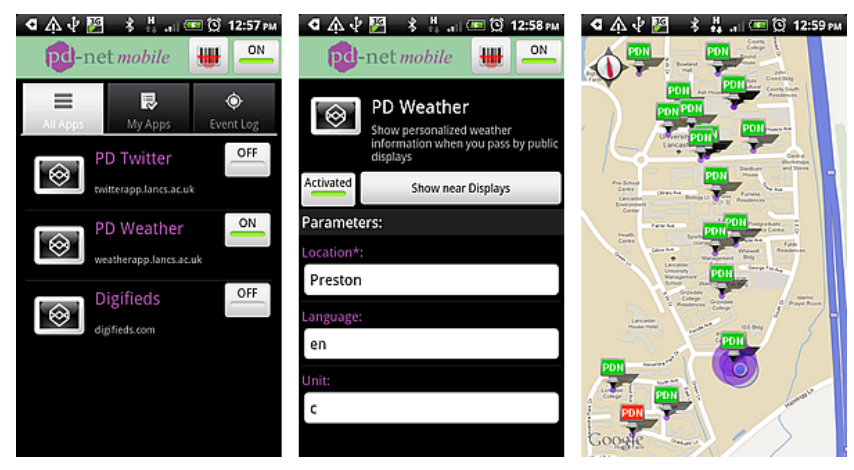

Figure 2: Mobile App UI

If a user's mobile application determines that they are in the vicinity of a public display that provides applications matching the users preferences (Figure 2), the mobile application contacts the relevant web-based display application, specifying the desired display and personalisation parameters. The web application will then contact the display component in order to ensure that the relevant content is shown on the display.

These applications have been tested as part of the Lancaster University e-Campus public display infrastructure.

\section{REQUIREMENTS}

Our demonstration requires power, two wireless network connections, wall space for a projection to mimic a public display and a small amount of table space to support a projector and associated computer.

\section{ACKNOWLEDGEMENTS}

The research leading to these results has received funding from the European Union Seventh Framework Programme (FP7/2007-2013) under grant agreement no. 244011.

\section{REFERENCES}

[1] Davies, N., Friday, A., Newman, P., Rutlidge, S., And Storz, O. Using Bluetooth Device Names to Support Interaction in Smart Environments. In Proc. of Mobisys '09 (2009).

[2] Sharifi, M., Payne, T., and David, E. Public display advertising based on bluetooth device presence. In Proc. of MIRW' 06 (2006). 